\title{
Why do some T cell receptor cytoplasmic domains associate with the plasma membrane?
}

\section{P. Anton van der Merwe ${ }^{1}{ }^{*}$, Hao Zhang ${ }^{1,2}$ and Shaun-Paul Cordoba ${ }^{1}$}

1 Sir William Dunn School of Pathology, University of Oxford, Oxford, UK

2 Dana-Faber Cancer Institute, Harvard Medical School, Boston, MA, USA

\author{
Edited by: \\ Oreste Acuto, University of Oxford \\ UK \\ Reviewed by: \\ Edward State Collins, The University \\ of North Carolina at Chapel Hill, USA \\ António Castro, University of Minho, \\ Portugal \\ ${ }^{*}$ Correspondence: \\ P. Anton van der Merwe, Sir William \\ Dunn School of Pathology, University \\ of Oxford, South Parks Road, Oxford \\ OX1 3RE, UK. \\ e-mail: anton.vandermerwe@ \\ path.ox.ac.uk
}

Based on studies in model systems it has been proposed that the cytoplasmic domains of $T$ cell receptor signaling subunits that have polybasic motifs associate with the plasma membrane, and that this regulates their phosphorylation. Recent experiments in more physiological systems have confirmed membrane association but raised questions as to its function.

Keywords: T cell receptor, membrane association, polybasic motifs, tyrosine phosphorylation
$\mathrm{T}$ cell triggering is initiated by the interaction of $\mathrm{T}$ cell receptor (TCR) with a cognate peptide presented by a major histocompatibility complex (pMHC) protein. The TCR complex consists of eight transmembrane proteins: TCR $\alpha \beta, \mathrm{CD} 3 \varepsilon \gamma, \mathrm{CD} 3 \varepsilon \delta$ heterodimers, and a TCR $\zeta$ homodimer (Call and Wucherpfennig, 2007). TCR $\alpha \beta$ binds pMHC and the remaining subunits transduce the signal, primarily through cytoplasmic immunoreceptor tyrosine-based activation motifs (ITAMs). Signal transduction requires phosphorylation of these ITAMs by the Src family tyrosine kinases Lck and/or Fyn. The phosphorylated ITAMs then recruit and activate the cytoplasmic zeta-chain-associated protein kinase 70 (Zap-70) through the association between doubly phosphorylated ITAMs and tandem SH2 domains on Zap-70 (Weiss and Littman, 1994).

The mechanism by which TCR engagement of pMHC leads to phosphorylation of TCR/CD3 ITAMs is still not well understood, and a number of models have been proposed (van der Merwe and Dushek, 2011). One hypothesis (Aivazian and Stern, 2000), recently termed the "safety" model (Kuhns and Davis, 2008), proposes that the cytoplasmic domains are protected from phosphorylation by sequestration of tyrosine residues at the plasma membrane. This model was first proposed when it was shown that the TCR $\zeta$ cytoplasmic domain peptide binds to anionic phospholipid vesicles, and this association inhibits phosphorylation in vitro by Src (Housden et al., 2003). Similar results have been

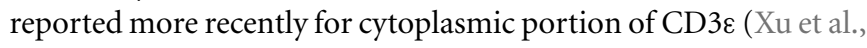
2008). In both cases this association in vitro was shown to be dependent on basic residue rich sequence (BRS/polybasic) motifs (Aivazian and Stern, 2000; Sigalov et al., 2006; Xu et al., 2008), consistent with an electrostatic interaction between anionic phospholipid headgroups and polybasic motifs. Analysis by circular dichroism and nuclear magnetic resonance spectroscopy suggested that membrane binding was accompanying by structural changes and/or burial of the tyrosines within the membranes (Aivazian and Stern, 2000; Xu et al., 2008). Fluorescent resonance energy transfer (FRET) was used to investigate plasma membrane association in intact cells (Xu et al., 2008). This confirmed that the cytoplasmic domain of $\mathrm{CD} 3 \varepsilon$ associates with the plasma membrane, and that this required polybasic motifs.

The "safety" model postulates that before TCR ligand engagement TCR $\zeta$ and CD3E ITAMs are sequestered on the plasma membrane to protect them from phosphorylation, and that TCR engagement results in their dissociation from the membrane to allow phosphorylation. Several recent studies have tested key predictions of the model. Two studies tested whether mutation of polybasic motifs that inhibited membrane association enhanced CD3E phosphorylation (DeFord-Watts et al., 2009; Fernandes et al., 2010). They found instead that, rather than enhancing phosphorylation, mutation of polybasic motifs reduced phosphorylation. Two subsequent studies focused on the TCR $\zeta$ cytoplasmic domain (DeFord-Watts et al., 2011; Zhang et al., 2011). Zhang et al. (2011) confirmed that the TCR $\zeta$ cytoplasmic domain associates with the plasma membrane though polybasic motifs, and went on to show that TCR/CD3 engagement is accompanied by its dissociation. However, this dissociation required, and was thus a consequence of, phosphorylation of TCRלITAMs (Zhang et al., 2011). Furthermore, mutation of polybasic motifs inhibited rather than enhanced TCR $\zeta$ phosphorylation and downstream signaling (DeFord-Watts et al., 2011; Zhang et al., 2011). Finally, inhibition of tyrosine phosphatase using pervanadate strongly induced phosphorylation and membrane dissociation of CD3 $\varepsilon$ and TCR $\zeta$ cytoplasmic domains in the absence of TCR ligand engagement (Fernandes et al., 2010; Zhang et al., 2011).

Thus, while these studies confirm that polybasic motifs mediate association of TCR $\zeta$ and $\mathrm{CD} 3 \varepsilon$ cytoplasmic domains with the plasma membrane, they imply that this association does not 


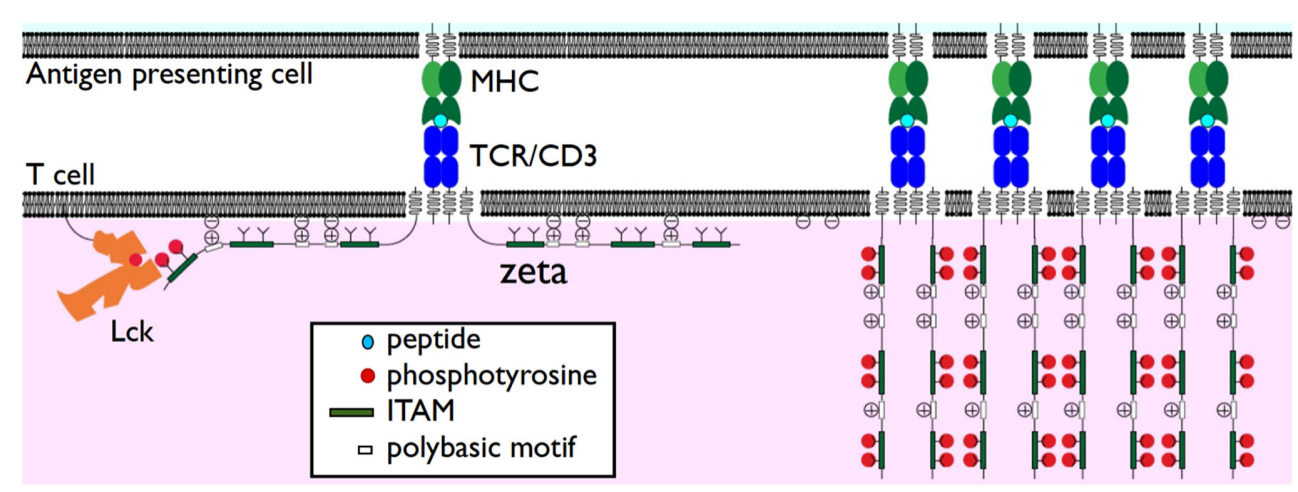

FIGURE 1 | Dissociation of TCR/CD3 cytoplasmic domains from the plasma membrane. The TCR $\zeta$ subunit cytoplasmic domains are shown associated with the plasma membrane in the resting state (left) through interactions of positively charged polybasic motifs and anionic phospholipids such as $\mathrm{PIP}_{2}$. Despite this membrane association TCR ITAMS are accessible to phosphorylation by Lck. Phosphorylation results in their dissociation from the plasma membrane (right). This may enhance TCR clustering and/or release sequestered phospholipids. prevent or even inhibit ITAM phosphorylation, contradicting the safety model. Indeed they suggest that this association may be required for optimal phosphorylation. How can we reconcile these findings with the evidence from previous in vitro studies (Aivazian and Stern, 2000; Xu et al., 2008) that ITAM tyrosine are buried in the membrane and protected from phosphorylation?

One explanation that has been proposed is that these in vitro studies may have been misleading (Sigalov and Hendricks, 2009). This is based on the observation that TCR $\zeta$ and CD $3 \varepsilon$ cytoplasmic domain peptides, like other polybasic peptides, can disrupt anionic phospholipid vesicles, producing potential artifacts (Sigalov and Hendricks, 2009).

Another possible explanation is that membrane association is very dynamic, allowing phosphorylation in the periods when ITAMs are not associated with the membrane. However, this does not explain the failure of the mutation of CD $3 \varepsilon$ and TCR $\zeta$ polybasic motifs, which decreases membrane association, to enhance ITAM phosphorylation. One proposed explanation for the latter result is that polybasic motif mutations, in addition to reversing membrane association, also directly disrupt the ability of Lck to bind to and/or phosphorylate ITAMs (Gagnon et al., 2010). Control experiments suggest that this may be the case for CD3 $\varepsilon$ (Gagnon et al., 2010), but not TCRל (Zhang et al., 2011).

Since recent experiments have failed to support the safety model they raise the question as to the functional significance of membrane association by TCR/CD3 cytoplasmic domains. We speculate here on two possible roles: prevention of spontaneous TCR/CD3 clustering and alteration of the lipid microenvironment. One consequence of having these additional interactions may be to decrease the mobility of the TCR/CD3 complex. In support of this, mutation of TCR/CD3 polybasic motifs does enhance TCR/CD3 mobility (Zhang et al., 2011). A second consequence could be to prevent homodimerization of TCR/CD3 cytoplasmic domains, as previously proposed (Sigalov et al., 2004). Collectively these two effects could help prevent spontaneous TCR/CD3 clustering in the absence of TCR triggering. It follows that TCR/CD3 ITAM phosphorylation following TCR triggering would facilitate TCR/CD3 clustering by inducing dissociation of these cytoplasmic domains from the membrane (Figure 1).
$\mathrm{T}$ cell receptor/CD3 polybasic motifs have been shown to associate particularly well with inositol phospholipids (DeFordWatts et al., 2009, 2011), including phosphatidylinositol 4,5bisphosphate $\left(\mathrm{PIP}_{2}\right)$, which have key roles in TCR/CD3 signal transduction. Studies in other systems (McLaughlin and Murray, 2005) suggest that this would have the effect of sequestering these phospholipids close to the TCR/CD3 complex and thus changing the TCR/CD3 lipid environment. This is supported by the observation that mutation of TCR $\zeta$ polybasic motifs alters the distribution of TCR/CD3 with respect to Lck (Zhang et al., 2011). One consequence of phosphorylation-induced membrane dissociation would be to make sequestered inositol phospholipids locally available for downstream signaling, for example as substrates for phospholipase C. Any such mechanism needs to account for the observation that mutation that eliminate binding of TCR $\zeta$ peptides to inositol phospholipids in vitro seem to have little effect TCR signal transduction (DeFord-Watts et al., 2011).

Investigation of the functional role of TCR/CD3 polybasic motifs will need to take into account the fact that there are many polybasic motifs in a single TCR/CD3 complex. Disruption of only a subset of these motifs may have only subtle effects. This could account for the modest effect of mutations of $\mathrm{CD} 3 \varepsilon$ polybasic motifs on $\mathrm{T}$ cell development and function in mice (DeFord-Watts et al., 2009).

In conclusion, while the available evidence suggests that the $\mathrm{TCR} \zeta$ and $\mathrm{CD} 3 \varepsilon$ cytoplasmic domains associate with the plasma membrane through polybasic motifs, and dissociate when phosphorylated, the functional role of membrane association remains unclear. The proposal, as in the "safety model" that it prevents ITAM phosphorylation is not supported by recent studies. Alternative models have been suggested but these have yet to be tested experimentally.

\section{ACKNOWLEDGMENTS}

We thank our fellow lab members for productive and stimulating discussions. P. Anton van der Merwe, Hao Zhang, and Shaun-Paul Cordoba are supported by Cancer Research UK, the UK Medical Research Council, and the EP Abraham Research Fund. 


\section{REFERENCES}

Aivazian, D., and Stern, L. J. (2000). Phosphorylation of $\mathrm{T}$ cell receptor $\zeta$ is regulated by a lipid dependent folding transition. Nat. Struct. Biol. 7, 1023-1026.

Call, M. E., and Wucherpfennig, K. W. (2007). Common themes in the assembly and architecture of activating immune receptors. Nat. Rev. Immunol. 7, 841-850.

DeFord-Watts, L. M., Dougall, D. S., Belkaya, S., Johnson, B. A., Eitson, J. L., Roybal, K. T., Barylko, B., Albanesi, J. P., Wülfing, C., and van Oers, N. S. (2011). The CD3 zeta subunit contains a phosphoinositide-binding motif that is required for the stable accumulation of TCR-CD3 complex at the immunological synapse. $J$. Immunol. 186, 6839-6847.

DeFord-Watts, L. M., Tassin, T. C., Becker, A. M., Medeiros, J. J., Albanesi, J. P., Love, P. E., Wülfing, C., and van Oers, N. S. (2009). The cytoplasmic tail of the $\mathrm{T}$ cell receptor $\mathrm{CD} 3$ subunit contains a phospholipid-binding motif that regulates $\mathrm{T}$ cell functions. J. Immunol. 183, 1055-1064.

Fernandes, R. A., Yu, C., Carmo, A. M., Evans, E. J., van der Merwe, P. A., and
Davis, S. J. (2010). What controls T cell receptor phosphorylation? Cell 142, 668-669.

Gagnon, E., Xu, C., Yang, W., Chu, H. H., Call, M. E., Chou, J. J., and Wucherpfennig, K. W. (2010). Response multilayered control of $\mathrm{T}$ cell receptor phosphorylation. Cell 142, 669-671.

Housden, H. R., Skipp, P. J., Crump, M. P., Broadbridge, R. J., Crabbe, T., Perry, M. J., and Gore, M. G. (2003). Investigation of the kinetics and order of tyrosine phosphorylation in the T-cell receptor zeta chain by the protein tyrosine kinase Lck. Eur. J. Biochem. 270, 2369-2376.

Kuhns, M. S., and Davis, M. M. (2008). The safety on the TCR trigger. Cell 135, 594-596.

McLaughlin, S., and Murray, D. (2005). Plasma membrane phosphoinositide organization by protein electrostatics. Nature 438, 605-611.

Sigalov, A., Aivazian, D., and Stern, L. (2004). Homooligomerization of the cytoplasmic domain of the $\mathrm{T}$ cell receptor zeta chain and of other proteins containing the immunoreceptor tyrosine-based activation motif. Biochemistry 43, 2049-2061.
Sigalov, A. B., Aivazian, D. A., Uversky, V. N., and Stern, L. J. (2006). Lipid-binding activity of intrinsically unstructured cytoplasmic domains of multichain immune recognition receptor signaling subunits. Biochemistry 45, 15731-15739.

Sigalov, A. B., and Hendricks, G. M. (2009). Membrane binding mode of intrinsically disordered cytoplasmic domains of $\mathrm{T}$ cell receptor signaling subunits depends on lipid composition. Biochem. Biophys. Res. Commun. 389, 388-393.

van der Merwe, P. A., and Dushek, O. (2011). Mechanisms for T cell receptor triggering. Nat. Rev. Immunol. 11, 47-55.

Weiss, A., and Littman, D. R. (1994). Signal transduction by lymphocyte antigen receptors. Cell 76, 263-274.

Xu, C., Gagnon, E., Call, M. E., Schnell, J. R., Schwieters, C. D., Carman, C. V., Chou, J. J., and Wucherpfennig, K. W. (2008). Regulation of $\mathrm{T}$ cell receptor activation by dynamic membrane binding of the $\mathrm{CD} 3$ epsilon cytoplasmic tyrosine-based motif. Cell 135, 702-713.

Zhang, H., Cordoba, S.-P., Dushek, O., and Anton van der Merwe, P.
(2011). Basic residues in the T-cell receptor $\zeta$ cytoplasmic domain mediate membrane association and modulate signaling. Proc. Natl. Acad. Sci. U.S.A. 108, 19323-19328.

Conflict of Interest Statement: The authors declare that the research was conducted in the absence of any commercial or financial relationships that could be construed as a potential conflict of interest.

Received: 20 January 2012; accepted: 10 February 2012; published online: 23 February 2012.

Citation: van der Merwe PA, Zhang $H$ and Cordoba S-P (2012) Why do some $T$ cell receptor cytoplasmic domains associate with the plasma membrane? Front. Immun. 3:29. doi: 10.3389/fimmu.2012.00029

This article was submitted to Frontiers in $T$ Cell Biology, a specialty of Frontiers in Immunology.

Copyright (C) 2012 van der Merwe, Zhang and Cordoba. This is an open-access article distributed under the terms of the Creative Commons Attribution Non Commercial License, which permits noncommercial use, distribution, and reproduction in other forums, provided the original authors and source are credited. 